\title{
Compact 5.2-GHz GaInP/GaAs HBT Gilbert Upconverter Using Lumped Rat-Race Hybrid and Current Combiner
}

\author{
Chinchun Meng, Member, IEEE, Tzung-Han Wu, and Mingchi Lin
}

\begin{abstract}
A compact 5.2-GHz Gibert upconversion mixer is demonstrated using the $2-\mu \mathrm{m}$ GaInP/GaAs heterojunction bipolar transistor (HBT) technology. A miniature lumped-element rat-race hybrid and an LC current combiner are used in the LO port and the RF port of the upconversion Gilbert mixer, respectively. The effect of the $\mathrm{LC}$ current combiner with inductor loss is established in this letter. An active IF balun is incorporated in the Gilbert upconverter with no extra power consumption. The fully integrated Gilbert upconverter has conversion gain of $1 \mathrm{~dB}, \mathrm{OP}_{1} \mathrm{~dB}$ of $-10 \mathrm{dBm}$, and $\mathrm{OIP}_{3}$ of $2 \mathrm{dBm}$ when input $\mathrm{IF}=300 \mathrm{MHz}, \mathrm{LO}=4.9 \mathrm{GHz}$, and output $\mathrm{RF}=\mathbf{5 . 2} \mathrm{GHz}$.
\end{abstract}

Index Terms-Balun, current mirror, GaInP/GaAs heterojunction bipolar transistor (HBT), Gilbert mixer, LC current combiner, lumped circuit, rat-race hybrid.

\section{INTRODUCTION}

D OUBLE balanced Gilbert mixers have small sizes and are widely used in RF IC designs. A Gilbert mixer needs balanced IF, LO, and RF signals for its optimum operation such as higher conversion gain, better dynamic range, and good isolation properties [1]. For a stand-alone Gilbert mixer IC, the balanced Gilbert mixer IF, LO, and RF ports are connected by the external baluns to the unbalanced board-level circuits. External baluns are bulky, expensive, inaccurate, and hard to use. The unbalanced-to-balanced and balanced-to-unbalanced conversions are still inevitable even for a fully differential transceiver. For example, most of the power amplifiers and pre-amplifiers are still in the single-ended configuration and thus an upconverter with a single-ended output is necessary. Sometimes it is beneficial to have an off-chip oscillator for better performance and a mixer with a single-ended LO port is needed. Active baluns have small sizes but consume extra power with the limited dynamic range [2]. However, it is difficult to incorporate the active baluns in the LO and RF ports because the active baluns have finite frequency responses. On the contrary, conventional distributed passive baluns eliminate these problems but occupy too much valuable real estate in the IC technology for the RF frequency range. A distributed rat-race hybrid balun can be miniaturized

Manuscript received February 11, 2005; revised May 27, 2005. This work was supported in part by the National Science Council of Taiwan, R.O.C. under Contracts NSC 93-2752-E-009-003-PAE and NSC 93-2219-E-009-026, by the Ministry of Economic Affairs under Contract 93-EC-17-A-05-S1-020, and by the National Chip Implementation Center (CIC). The review of this letter was arranged by Associate Editor F. Ellinger.

The authors are with the Department of Communication Engineering, National Chiao Tung University, Hsinchu 300, Taiwan, R.O.C. (e-mail: ccmeng@ mail.nctu.edu.tw).

Digital Object Identifier 10.1109/LMWC.2005.856844

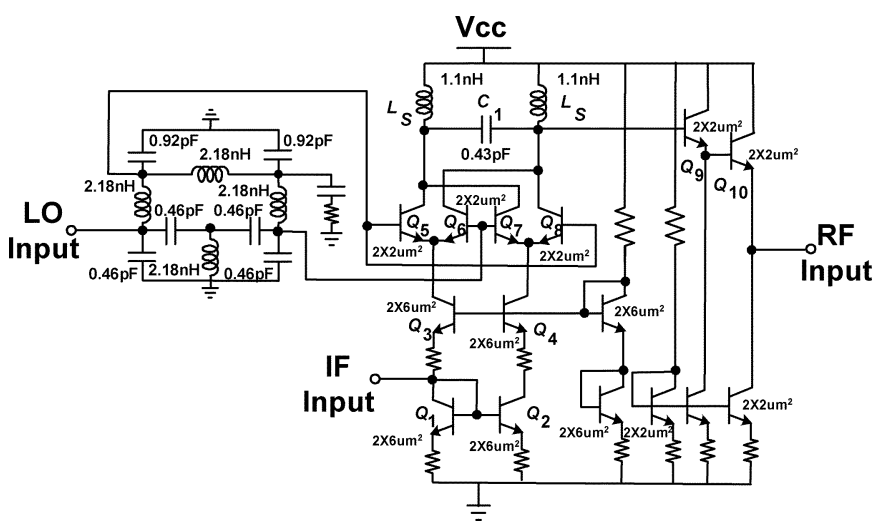

Fig. 1. Schematic diagram of the GaInP/GaAs HBT Gilbert upconverter with the lumped rat-race hybrid and the LC current combiner.

by using its lumped equivalence [3]. A passive LC current combiner can double the output current and converts the differential outputs into a single-ended output [4]. GaAs material has the semi-insulating substrate and thus good quality lumped-element inductors when compared with the silicon technology. The goldplated backside ground in GaAs technology also makes the IC compatible with the unbalanced microstrip line structure. Thus, it is our objective in this letter to demonstrate a compact RF Gilbert upconversion mixer requiring no external baluns using GaInP/GaAs HBT technology. The LC passive combiner provides 6-dB gain improvement when compared with the singleended output. The upconverter preserves the truly balanced operation of the Gilbert mixer while the single-ended terminals are compatible with the unbalanced microstrip line structures of GaAs RF ICs and board-level circuits.

\section{Circuit Design}

A fully integrated GaInP/GaAs HBT Gilbert upconversion mixer is shown in Fig. 1. The upconversion mixer in Fig. 1 consists of a LO Gilbert mixer core $\left(Q_{5}, Q_{6}, Q_{7}\right.$, and $\left.Q_{8}\right)$ with a lumped $180^{\circ}$ rat-race hybrid, an active IF balun $\left(Q_{1}, Q_{2}\right.$, $Q_{3}$, and $\left.Q_{4}\right)$, and an RF output LC current combiner with a Darlington common-collector output buffer $\left(Q_{9}\right.$ and $\left.Q_{10}\right)$. The lumped rat-race hybrid shown in Fig. 1 employs the pi low-pass network and the tee high-pass network to replace the quarterwavelength and three-quarter-wavelength sections of the distributed counterparts, respectively [3]. The lumped rat-race hybrid generates balanced LO signals for the mixer core. The LC current combiner formed by two inductors $L_{S}$ and one capacitor $\mathrm{C}_{1}$ as shown in Fig. 1 is located at the RF output of the 


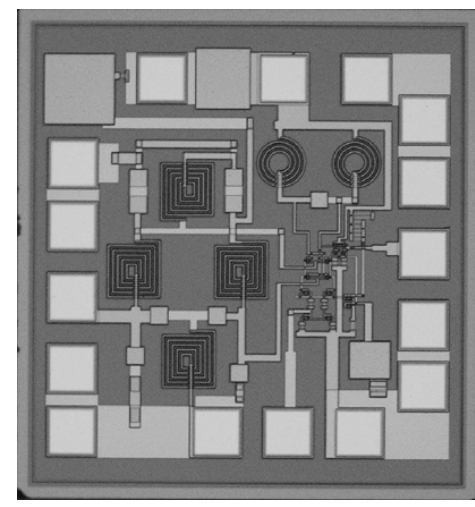

Fig. 2. Photograph of the GaInP/GaAs HBT Gilbert upconverter with the lumped rat-race hybrid and the LC current combiner.

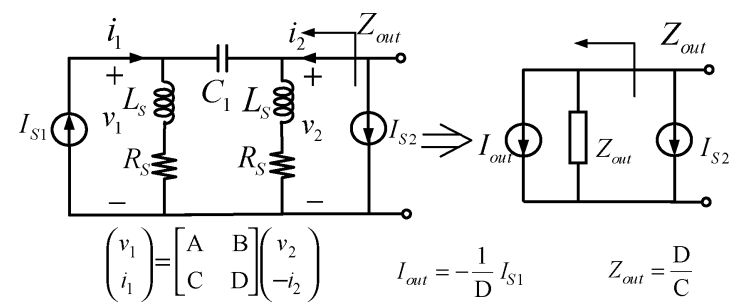

Fig. 3. LC current combiner operational principle. The inductor loss is included in the analysis.

Gilbert mixer core to perform the differential-to-single conversion. A Darlington common collector output follows the current combiner to facilitate impedance matching at the RF port. The common-base-configured $Q_{3}$ and common-emitter-configured $Q_{2}$ form the active IF balun by providing equal but out of phase transconductance gain when $Q_{1}$ and $Q_{2}$ are connected as a current mirror [5]. The single-to-differential transconductor replaces the conventional emitter-coupled-pair transconductor in the Gilbert upconverter and does not consume extra power [4], [6].

The photograph of the upconverter is shown in Fig. 2. The die size is $1 \mathrm{~mm}^{2}$. The $2-\mu \mathrm{m} \mathrm{GaInP/GaAs} \mathrm{HBT} \mathrm{technology} \mathrm{has} \mathrm{a}$ peak $\mathrm{f}_{\mathrm{T}}$ around $40 \mathrm{GHz} .2 \times 2 \mu \mathrm{m}^{2}$ single-emitter HBT devices are used throughout most of the circuits except for the IF active balun and diode-connected current-mirror transistors that use the $2 \times 6 \mu \mathrm{m}^{2}$ single-emitter HBT devices. Four 5.5-square-turn $(2.18 \mathrm{nH})$ inductors are employed in the rat-race hybrid and two 3.5-circular-turn $(1.1 \mathrm{nH})$ inductors are employed in the LC current combiner, respectively.

\section{ANALYSIS OF THE LC CURRENT COMBINER WITH INDUCTOR LOSS}

The detailed operational principle of the LC current combiner with inductor loss is explained in Fig. 3. $I_{S 1}$ equals to $I_{S 2}$ in Fig. 3 for the differential excitation of the current combiner. The output resistance looking into the collector of a GaInP/GaAs HBT device is very high because the GaInP/GaAs HBT device has a heavily-doped base and thus a very high Early voltage. Thus, no resistor in parallel with the differential excitation is needed in Fig. 3. The inductor model employed here consists of an inductance $L_{S}$ in series with a parasitic resistor $R_{S}$. The unwanted parasitic capacitance of an on-chip inductor sets the practical upper frequency limit for the simple inductor model to be effective. GaAs has less parasitic capacitance because of the semi-insulating substrate and thus on-chip inductors with higher quality factors are available at high frequencies. The combination of the LC current combiner and the current source $I_{S 1}$ can be represented by its Norton equivalence $I_{\text {out }}$ and $Z_{\text {out }}$ in Fig. 3 . The $I_{\text {out }}$ and $\mathrm{Z}_{\text {out }}$ can be related to the ABCD matrix elements of the LC current combiner as shown in Fig. 3.

The ABCD matrix of the LC current combiner obtained by cascading the $\mathrm{ABCD}$ matrix of the shunt inductors and the series capacitor is

$$
\left[\begin{array}{ll}
\mathrm{A} & \mathrm{B} \\
\mathrm{C} & \mathrm{D}
\end{array}\right]=\left[\begin{array}{cc}
\frac{R_{S}+j \omega L_{S}+\frac{1}{j C_{1}}}{R_{S}+j \omega L_{S}} & \frac{1}{j \omega C_{1}} \\
\frac{2\left(R_{S}+j \omega L_{S}\right)+\frac{1}{j C_{1}}}{\left(R_{S}+j \omega L_{S}\right)^{2}} & \frac{R_{S}+j \omega L_{S}+\frac{1}{j \omega C_{1}}}{R_{S}+j \omega L_{S}}
\end{array}\right] .
$$

The effect of the current doubling occurs at the resonant frequency, $\omega_{R}$. Here

$$
\omega_{R}=\sqrt{\frac{1}{2 L_{S} C_{1}}} .
$$

Thus, $I_{\text {out }}$ and $\mathrm{Z}_{\text {out }}$ at the resonant frequency can be expressed as follows:

$$
\begin{aligned}
I_{\text {out }} & =-\frac{R_{S}+j \omega_{R} L_{S}}{R_{S}-j \omega_{R} L_{S}} I_{S 1} \\
Z_{\text {out }} & =\frac{R_{S}^{2}+\left(\omega_{R} L_{S}\right)^{2}}{2 R_{S}}=\frac{R_{S}\left(1+Q_{R}^{2}\right)}{2}=\frac{R_{\mathrm{P}}}{2} .
\end{aligned}
$$

Here, $Q_{R}$ in (4) is the quality factor of the inductor at the resonant frequency. $R_{\mathrm{P}}$ in (4) can be interpreted as the parallel resistor when the series inductor model with $L_{S}$ and $R_{S}$ is replaced by the parallel inductor model with $L_{\mathrm{P}}$ and $R_{\mathrm{P}}$. The output resistance is simply expressed as $R_{\mathrm{P}} / 2$. If the quality factor $Q_{R}$ is much larger than one, $I_{\text {out }}$ equals to $I_{S 1}$ and $L_{\mathrm{P}}$ equals to $L_{S}$. Thus, the voltage at the output node of the LC current combiner equals to $I_{S 1} R_{\mathrm{P}}$ for a differential excitation because of the current doubling effect.

It is straightforward that the voltage gain formula for a differential amplifier with the LC current combiner at the resonant frequency is exactly the same as the well-known voltage gain formula for a differential amplifier with the active-current-mirror load. Only the output resistance of the active current mirror is replaced by the $R_{\mathrm{P}}$ in the case of the LC current combiner. It is advantageous to employ the passive LC current combiner in upconverter applications because the LC combiner eliminates the slow response associated with the active-current-mirror load and is very compact in size.

The RF 3-dB bandwidth and IF 3-dB bandwidth are determined by the RF LC current combiner and the LO lumped ratrace hybrid, respectively. The simulated RF $3-\mathrm{dB}$ bandwidth is $280 \mathrm{MHz}$ by fixing the $\mathrm{LO}$ frequency at $4.9 \mathrm{GHz}$ and sweeping the IF frequency. The simulated IF 3-dB bandwidth is $140 \mathrm{MHz}$ by fixing the RF frequency at $5.2 \mathrm{GHz}$ and sweeping the IF frequency. 


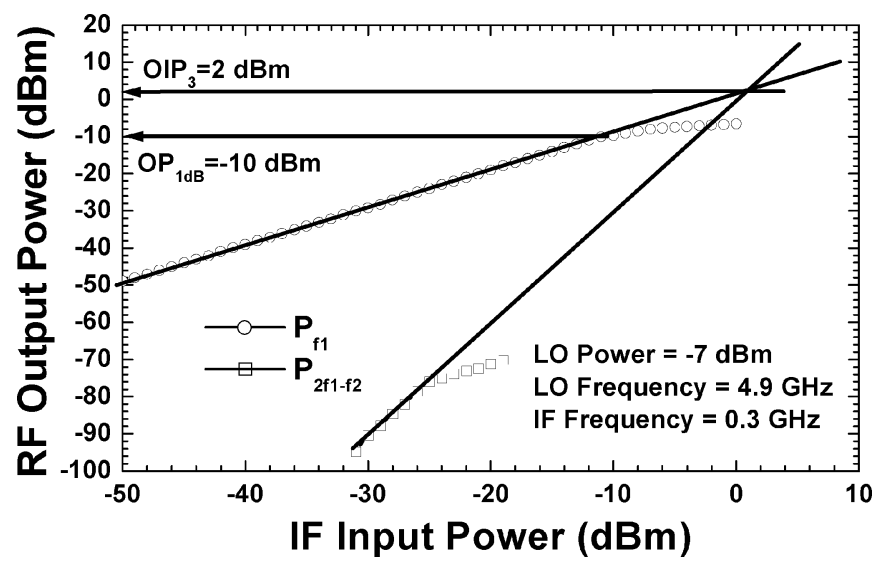

Fig. 4. Power performance of the GaInP/GaAs HBT Gilbert upconverter with the lumped rat-race hybrid and the LC current combiner.

TABLE I

COMPARISON OF THE UP-CONVERSION GILBERT MIXERS

\begin{tabular}{|c|c|c|c|c|}
\hline & This Work & $\operatorname{Ref}[1]$ & $\operatorname{Ref[7]}$ & $\operatorname{Ref}[8]$ \\
\hline RF (GHz) & 5.2 & 20 & 1.9 & 12 \\
\hline Gain $(\mathrm{dB})$ & 1 & $15.3^{*}$ & -6.7 & -2 \\
\hline $\begin{array}{l}\text { LO-RF } \\
\text { Isolation }(\mathrm{dB})\end{array}$ & 38 & 32 & N/A & 22 \\
\hline $\mathrm{OP}_{1 \mathrm{~dB}}(\mathrm{dBm})$ & -10 & -1 & $-3-$ & $13.5^{* *}$ \\
\hline $\mathrm{OIP}_{3}(\mathrm{dBm})$ & 2 & 6 & 2 & $2 * *$ \\
\hline $\begin{array}{l}\text { Mixer Core } \\
\text { Current (mA) }\end{array}$ & 2.3 & 12 & 15 & 16 \\
\hline Technology & $\begin{array}{c}2 \text { um } \\
\text { GaInP/ } \\
\text { GaAs HBT }\end{array}$ & $\begin{array}{l}\text { lum } \\
\text { InP } \\
\text { SHBT }\end{array}$ & $\begin{array}{c}\text { 2um } \\
\text { GaInP/ } \\
\text { GaAs HBT }\end{array}$ & $\begin{array}{l}\text { lum } \\
\text { InP } \\
\text { SHBT }\end{array}$ \\
\hline
\end{tabular}

\section{MeAsured Performance}

The GaInP/GaAs HBT Gilbert upconverter with single-ended IF, LO, and RF ports facilitates on-wafer rf measurements. The supply voltage is $5 \mathrm{~V}$ and the current consumption is $6.5 \mathrm{~mA}$. The measured peak conversion gain is $1 \mathrm{~dB}$ when LO power is $-7 \mathrm{dBm}$ and LO frequency is $4.9 \mathrm{GHz}$. The measured conversion gain varies within $1 \mathrm{~dB}$ when $L O$ power changes from $-12 \mathrm{dBm}$ to $-1 \mathrm{dBm}$. The output $\mathrm{RF}$ return loss is $23 \mathrm{~dB}$ at $5.2 \mathrm{GHz}$ and the IF input return loss is better than $25 \mathrm{~dB}$ for frequencies up to $8 \mathrm{GHz}$. The power performance of the upconverter is shown in the Fig. 4. The fully integrated Gilbert upconverter has conversion gain of $1 \mathrm{~dB}, \mathrm{OP}_{1} \mathrm{~dB}$ of $-10 \mathrm{dBm}$, and $\mathrm{OIP}_{3}$ of $2 \mathrm{dBm}$ when input IF $=300 \mathrm{MHz}, \mathrm{LO}=4.9 \mathrm{GHz}$ and output $\mathrm{RF}=5.2 \mathrm{GHz}$. The measured LO-RF isolation is $38 \mathrm{~dB}$.

In order to compare the performance of the upconversion Gilbert mixers, the state-of-the-art GaAs HBT and InP single heterojunction bipolar transistor (SHBT) Gilbert upconverters are summarized in Table I [7], [8]. The InP SHBT upconverters have better frequency response because of the higher cutoff frequency. The GaInP/GaAs HBT Gilbert upconverter with LC current combiner in this work has the best LO-RF isolation and the smallest Gilbert mixer core current consumption. The difference between $\mathrm{OIP}_{3}$ and $\mathrm{OP}_{1} \mathrm{~dB}$ can be used to indicate the linearity and our work also shows good linearity. The linearity can be further improved by increasing the current through the Gilbert cell. In other words, our work shows the best performance in terms of the combinations of linearity and current consumption.

\section{CONCLUSION}

A 5.2-GHz GaInP/GaAs HBT Gilbert upconversion mixer with on-chip baluns is demonstrated in this letter. A truly balanced operation is preserved in the fully integrated $\mathrm{GaInP} / \mathrm{GaAs}$ HBT Gilbert upconverter with single-ended IF, LO, and RF ports. A miniature lumped-element rat-race hybrid and an LC current combiner are used in the LO port and the RF port of the 5.2-GHz GaInP/GaAs HBT upconversion Gilbert mixer, respectively. A theoretical analysis for the LC current combiner with inductor loss is also established. The deep submicron CMOS devices possess excellent cut-off frequencies and on-chip inductors with high quality factors are feasible in the standard CMOS process [9]. Thus, the design principle demonstrated in this letter can also be realized on the industrial CMOS technology.

\section{REFERENCES}

[1] K. W. Kobayashi, R. M. Desrosiers, A. G. -Aitken, J. C. Cowles, B. Tang, L. T. Tran, T. R. Block, A. K. Oki, and D. C. Streit, "A DC-20-GHz InP HBT balanced analog multiplier for high-data-rate direct-digital modulation and fiber-optic receiver applications," IEEE Trans. Microw. Theory Tech., vol. 48, no. 2, pp. 194-202, Feb. 2000.

[2] H. Ma, S. J. Fang, F. Lin, K.-S. Tan, J. Shibata, A. Tamura, and H. Nakamura, "A GaAs upconveretr MMIC with an automatic gain control amplifier for 1.9 GHz PHS," IEEE J. Solid-State Circuits, vol. 33, no. 9, pp. 1297-1305, Sep. 1998.

[3] S. J. Parisi, " $180^{\circ}$ lumped element hybrid," in IEEE MTT-S Int. Dig., 1989, pp. $1243-1246$.

[4] C. C. Meng, S. K. Hsu, A. S. Peng, S. Y. Wen, and G. W. Huang, "A fully integrated $5.2 \mathrm{GHz}$ GaInP/GaAs HBT upconversion micromixer with output LC current combiner and oscillator," in IEEE MTT-S Int. Dig., 2003, pp. A205-A208.

[5] J. Durec and E. Main, "A linear class AB single-ended to differential transconverter suitable for RF circuits," in IEEE MTT-S Dig., 1996, pp. 1071-1074.

[6] B. Gilbert, "The MICROMIXER: A highly linear variant of the Gilbert mixer using a bisymmetric class-AB input stage," IEEE J. Solid-State Circuits, vol. 32, no. 9, pp. 1412-1423, Sep. 1997.

[7] J. M. Wu, J. K. Jau, T. S. Horng, and C. C. Tu, "Highly linear upconversion MMIC designs with complete package and test board effects for CDMA applications," in Proc. IEEE Radio Frequency Integrated Circuit Symp., 2003, pp. 405-408.

[8] K. W. Kobayashi, L. T. Tran, S. Bui, A. K. Oki, D. C. Streit, and M. Rosen, "InAlAs/InGaAs HBT X-band double-balanced upconverter," IEEE, J. Solid-State Circuits, vol. 29, no. 10, pp. 1238-1242, Oct. 1994.

[9] L. F. Tiemeijer, R. J. Havens, N. Pavlovic, and D. M. W. Leenaerts, "Record $Q$ symmetrical inductors for 10-GHz LC-VCOS in $0.18-\mu \mathrm{m}$ gate-length CMOS," IEEE Electron Device Lett., vol. 23, no. 12, pp. 713-715, Dec. 2002. 